Einführung in das Schwerpunktthema

\title{
Rückbindung der Wirtschaft an die Naturräume
}

\section{Von Alexandra Dehnhardt und Ulrich Petschow}

ie im Jahr 2000 verabschiedete Wasserrahmenrichtlinie der Europäischen Union befindet sich gegenwärtig in der Umsetzungsphase (vgl. dazu näher den Beitrag von Brackemann et al.). Wesentliche Ziele der Richtlinie sind

1. Ansätze des integrierten Managements in einem naturräumlichen Kontext - dem Flusseinzugsgebiet - und

2. ein guter ökologischer Zustand der Gewässer - von einigen zum Teil weit reichenden Ausnahmen abgesehen.

Mit der Wasserrahmenrichtlinie (WRRL) sind gerade auch für Deutschland erhebliche Veränderungen für die Bewirtschaftungsplanung verbunden:

- Der nunmehr geschaffene Bezugspunkt zum Flusseinzugsgebiet soll Fragmentierungen überwinden, die sich aufgrund von Ländergrenzen und damit der Inkompatibilität von Verwaltungs- und naturräumlichen Strukturen ergeben. Im Grundsatz werden damit Bioregionen konstruiert - hier in Bezug auf Wasserressourcen. Deren nachhaltiges Management erfordert, dass das Gesamtsystem mit seinen sozialen und ökonomischen Nutzungen im Hinblick auf den Umgang mit den Wasserressourcen optimiert werden muss.

- Gleichzeitig geht es um die Überwindung des bisherigen command and control-Ansatzes, der nicht zuletzt zu ökonomischen Ineffizienzen beiträgt. Beispielsweise sind Maßnahmen zur Verminderung der diffusen Belastungen, die zunehmend an Bedeutung gewinnen, mit einem solchen Ansatz nicht hinreichend steuerbar.

- Daneben stellt der Aspekt der Partizipation als genereller Grundsatz im Rahmen der WRRL einen neuen Ansatz für die Bewirtschaftungsplanung dar. Die wesentlichen Fragen stellen sich dabei in Bezug auf die Partizipationsmöglichkeiten - Institutionalisierung, Entscheidungsstrukturen, einbezogene Akteure - und ob ihre Erweiterung geeignet sein kann, zu einer kontinuierlichen Verbesserung des Gewässerzustandes beizutragen.

Obwohl integrierte Managementansätze auch für Deutschland nicht vollkommen neu sind, stellt die WRRL mit den skizzierten Anforderungen eine er- hebliche Herausforderung für das institutionelle Gefüge im Wassermanagement dar. Dies gilt nicht allein für die zu entwickelnden Strukturen und die anspruchsvollen zeitlichen Fristen, sondern auch im Hinblick auf die ökonomische Analyse sowie die Ausgestaltung, Möglichkeiten und Grenzen der Partizipation im Sinne der WRRL (1).

\section{Die Beiträge im Überblick}

Im Rahmen dieses Schwerpunktes haben wir sechs Beiträge versammelt, die aus unterschiedlicher Sichtweise das Flusseinzugsgebietmanagement beleuchten, nämlich aus der Sicht der 1. Prozesse und der Implementation,

2. umweltpolitischen Instrumentendebatte

3. US-amerikanischen Erfahrungen,

4. französischen Erfahrungen,

5. Modelle und der Einbeziehung der Akteure und schließlich der

6. ökologisch-ökonomischen Bewertung.

Brackemann, Markard und Rechenberg geben zunächst einen Überblick über die Prozesse der Implementierung der WRRL sowohl auf der EUEbene als auch auf der deutschen Ebene und verweisen auf die besonderen Probleme, die gerade in einem föderalen Staat auftreten. Petschow stellt neue Ansätze der Umweltpolitik dar und sieht dabei die WRRL als Beispiel des Ökosystemmanagements an. Dabei geht es erstens um die Notwendigkeit eines adaptiven Managements im Kontext von Natur als dynamischem System und zweitens um die Frage der Beteiligungs- und Entscheidungsverfahren im Umgang mit den Wasserressourcen. Diese können als common pool resources aufgefasst werden und müssen damit keineswegs der Tragödie der Allmende anheim fallen; vielmehr sind auch vor Ort Nutzungsregeln entwickelbar.

Zwei Beiträge beschäftigen sich danach mit Erfahrungen anderer Länder: Genskow und Born fassen die US-amerikanischen Erfahrungen mit dem watershed management zusammen, das mittlerweile - mit unterschiedlichen Erfolgen - seit über zehn Jahren praktiziert wird. Sie kommen zu einer positiven Einschätzung dieses Ansatzes, mit dem es teilweise gelungen ist, komplexe Umweltprobleme im Flusseinzugsgebiet Lösungen zuzuführen und dabei eine eigene Dynamik zu entfalten. Bongaerts stellt die Situation in Frankreich dar, wo die Umsetzung der WRRL zumindest bezüglich der institutionellen Strukturen ein geringes Problem darstellt, da der Ansatz im Grundsatz bereits existierte. Daneben bestehen auch bereits Strukturen, die eine Partizipation ermöglichen sollen. Gleichwohl bleibt als offene Frage, inwieweit sich gerade diese Strukturen als geeignet erweisen, zu ökologischen Fortschritten beizutragen.

Um die komplexen ökologischen Beziehungen im Flusseinzugsgebiet zu erfassen und auch für partizipative Prozesse nutzbar zu machen, werden zunehmend Modelle und Bewertungsverfahren eingesetzt. Welp betont in seinem Beitrag, dass Modelle geeignet sein können, partizipative Prozesse aber vor allem auch das Problemverständnis zu verbessern. In diesem Sinne kann die Modellierung einen wesentlichen Beitrag zum integrierten Flusseinzugsgebietmanagement leisten. Dehnhardt befasst sich schließlich mit der Frage der ökonomischen Bewertung und geht dabei auf die Ansätze der WRRL ein. Diese sind im Wesentlichen kostenorientiert, wenngleich der teilweise vorgesehene Einbezug externer Kosten zumindest im Grundsatz eine erhebliche Erweiterung der bisherigen Ansätze darstellt. In einem zweiten Schritt wird eine Systematisierung der möglichen Ansätze zur Erfassung der Nutzenkomponenten von Maßnahmen im Flusseinzugsgebiet vorgenommen. Dies stellt nicht nur im Kontext der WRRL eine bislang weitgehend vernachlässigte Ebene dar, deren Entwicklung gerade für eine bessere Abbildung der ökologischenökonomischen Vorteile einer stärkeren Rückbindung der Wirtschaft an die Naturräume von Bedeutung ist.

\section{Anmerkung}

(1) Das Institut für ökologische Wirtschaftsforschung ist derzeit in zwei modellgestützte BMBF-Forschungsvorhaben zum Flusseinzugsgebietsmanagement eingebunden, wobei die IÖW-Beiträge insbesondere auf Fragen der ökonomischen Bewertung sowie der Partizipation orientiert sind.

\section{Die Autorlnnen}

Alexandra Dehnhardt und Ulrich Petschow sind wissenschaftliche MitarbeiterInnen im Forschungsfeld Umweltökonomie und -politik des Instituts für ökologische Wirtschaftsforschung (IÖW).

Kontakt: IÖW, Potsdamer Str. 105, 10785 Berlin.

Tel. 030/884594-0, Fax 030/ 8825439,

E-mail: Alexandra.Dehnhardt@ioew.de, Ulrich.Petschow@ioew.de 
(c) 20I0 Authors; licensee IÖW and oekom verlag. This is an article distributed under the terms of the Creative Commons Attribution Non-Commercial No Derivates License (http://creativecommons.org/licenses/by-nc-nd/3.o/), which permits unrestricted use, distribution, and reproduction in any medium, provided the original work is properly cited. 